Retrieval of these stents can be challenging and requires utilisation of various endoscopic grasping devices. With increasing use of ERCP and biliary stents this is being encountered more frequently. Most stents can be retrieved with conventional grasping devices like stent grabbers or biopsy forceps. However, some case may require the use of novel through the cholangioscope devices.

We present a 37-year-old lady who presented with choledocholithiasis and 4 conventional ERCPs were not successful in clearing the CBD stones. At spy glass cholangiscopy we discovered a proximally migrated biliary stent. This video demonstrates a novel technique to retrieve the migrated biliary stent.

\section{PTH-067 TREATMENT OF BLUE RUBBER BLEB NEVUS SYNDROME IN A PAEDIATRIC PATIENT ASSISTED BY DOUBLE- BALLOON ENTEROSCOPE}

${ }^{1}$ Nikolaos Lazaridis* , Alberto Murino, ${ }^{1}$ Nikolaos Koukias, ${ }^{1}$ Deborah Costa, ${ }^{1}$ Andrea Telese, ${ }^{1}$ Claudia Coppo, ${ }^{1}$ Regina Raymond, ${ }^{2}$ Daniel Crespi, ${ }^{2}$ Mark Furman, ${ }^{1}$ Edward J Despott. ${ }^{1}$ The Royal Free Unit for Endoscopy, The Royal Free Hospital and University College London (UCL) Institute For Liver and Digestive Health, London, UK; ${ }^{2}$ Centre for Paediatric Gastroenterology, The Royal Free Hospital and University College London (UCL) Institute for Liver and Digestive Health, London, UK

\subsection{6/gutjnl-2019-BSGAbstracts.92}

Introduction Blue rubber bleb nevus syndrome (BRBNS) is an extremely rare systemic vascular disorder characterised by multiple cutaneous and gastrointestinal venous malformations. Patients present with fatigue, iron deficiency anaemia (IDA) and occult or overt gastrointestinal (GI) bleeding. Patients are usually treated with conservative management including iron supplementation and blood transfusions. However, endoscopic (argon plasma coagulation, sclerotherapy, polypectomy, band ligation etc), radiological and surgical approaches are preferred for severe cases.

Aims and Methods A 7-year-old female patient with iron deficiency anaemia and multiple cutaneous lesions was diagnosed with BRBNS at a local hospital. The patient was referred to our institution for further management due to blood transfusions dependence and PR bleeding. A small bowel capsule endoscopy (SBCE) revealed two vascular lesions in the small bowel.

Results An anterograde double-balloon enteroscopy (DBE) was the performed under general anaesthesia. Two $20 \mathrm{~mm}$ vascular lesions were identified in the gastric body. A loop ligating device (Olympus, Tokyo, Japan) was applied around the base of each lesion then tightened and completely detached. No further vascular malformations were found in the duodenum, jejunum and proximal ileum. Although the number of units of blood transfusion decreased over the next 6 months a followup retrograde DBE was performed due to persistent anaemia. Six lesions were identified in the transverse colon (2), caecum (1) and distal ileum (3). Ligation loop was used for 2 colonic lesions while two ileac rubber blub lesions were treated with both ligation loop and metallic clips. Since the 2 remaining lesions were flat and floppy, loop ligation was not technically feasible. No immediate and post procedural complications (including delayed bleeding) occurred.

Conclusion DBE facilitated loop ligation appears to be a safe and minimally invasive option in patients affected by BRBNS reducing the blood transfusion dependence.

\section{PTH-068 SPORADIC LATERALLY SPREADING NONAMPULLARY DUODENAL ADENOMAS: THE ROLE OF SALINE- IMMERSION THERAPEUTIC ENDOSCOPY}

Nikolaos Lazaridis*, Alberto Murino, Nikolaos Koukias, Andrea Telese, Deborah Costa, Claudia Coppo, Regina Raymond, Edward J Despott. The Royal Free Unit for Endoscopy, The Royal Free Hospital and University College London (UCL) Institute for Liver and Digestive Health, London, UK

\subsection{6/gutjnl-2019-BSGAbstracts.93}

Introduction Sporadic laterally spreading nonampullary duodenal adenomas (SLSNDA) are an uncommon incidental finding during oesophagogastroduodenoscopy. Endoscopic mucosal resection (EMR) in the duodenum is challenging due to increased risk of perforation and bleeding. Underwater EMR (UEMR) is a novel and effective endoscopic resection technique performed without submucosal injection. Saline-immersion therapeutic endoscopy (SITE) is an evolution of UEMR and saline solution is used instead of water to minimise the risk of water intoxication.

Aims and methods Our aim was to evaluate the efficacy and safety of SITE-EMR for SLSNDA via a retrospective review of SLSNDA resected by SITE-EMR at our institution from May 2017 to October 2018. Demographic, clinical, endoscopic findings and follow-up data were analysed.

Results

Nine SLSNDA (median size $25 \mathrm{~mm}$ ) were found in eight patients (4 male, median age: 69 year-old). One was located in D1, 4 in D2 and 4 in D3. En-bloc resection was achieved in two lesions (23\%) while wide-field resection was performed in seven lesions (77\%). Complete resection was achieved in seven patients $(87.5 \%)$. A circumferential lesion involving the whole duodenal bulb was found in one case and SITE-EMR technique was not feasible as well as other alternative endoscopic resection techniques due to severe fibrosis; the patient was therefore referred for surgery and excluded from further analysis. Histological results revealed six (75\%) tubulo-villous adenomas with low-grade dysplasia and two tubular adenomas low-grade dysplasia (25\%). Immediate complications including perforation and bleeding did not occur. One patient (12.5\%) presented with delayed GI bleeding 24hours post procedure and was treated successfully with endoclips. Three cases $(37.5 \%)$ of recurrences were identified at 3 months follow-up requiring further endoscopic treatment. No further recurrence was identified at 6 and 12 months follow-up in any patient.

Conclusion SITE-EMR of SLSNDA appears to be a safe and effective management with low recurrence rates at long term follow-up.

\section{PTH-069 SIMULATED CASES; A TOOL TO TEACH ENDOSCOPY NON-TECHNICAL SKILLS}

${ }^{1}$ Louise Macdougall*, 'Peter Coyne, ${ }^{2}$ Mike Bradburn, ${ }^{1}$ Christopher Mountford. ${ }^{1}$ Newcastle upon Tyne Hospitals NHSFT, Newcastle-upon-tyne, UK; ${ }^{2}$ Northumbria Healthcare NHSFT, Newcastle-upon-tyne, UK

\subsection{6/gutjnl-2019-BSGAbstracts.94}

Introduction There has been growing awareness of the importance of non-technical skills (NTS) in endoscopy however training in this area varies and may not be explicit in many departments. It is important that this is recognised and efforts are made to improve awareness. In the Northern Deanery the REST (regional endoscopic skills training) course has been 
developed for gastroenterology and surgical trainees and consists of a series of one day training events spread over several months to supplement departmental colonoscopy training and uses a variety of classroom based and simulation training. To facilitate the teaching of NTS we developed a video of a simulated case to form the basis of discussion.

Methods A video was made of a simulated case that could be used to highlight NTS. As the attached video demonstrates the video highlighted a case where the use of non-technical skills could be optimised. This was used as a starting point for discussion with delegates.

Pre and post course confidence ratings were collected on a variety of areas including human factors, mean ratings were calculated and mann-whitney $\mathrm{U}$ test applied.

Results Eight candidates completed the course, four surgical and four gastroenterology trainees. Six had already the JAG basic skills course. Confidence rating in human factors improved from a mean of 5.9 (out of 10) to 8.12 (p 0.03)

Conclusions Emphasis on non-technical skills training is imperative to improve and ensure patient safety and minimise adverse events. The teaching of non-technical skills may not always be explicit within working departments. Whilst simulation can be a useful teaching tool for NTS it can be time consuming This video demonstrates a resource-low method by which this could be taught as part of a wider endoscopy course. Whilst we only measured self ratings rather than assessing performance trainees confidence rating in human factors significantly improved suggesting this is a useful teaching tool.

\section{PTH-070 SEVERE MIDGUT BLEEDING FROM JEJUNAL DIVERTICULA TREATED BY DOUBLE-BALLOON ENTEROSCOPY}

${ }^{1}$ Alberto Murino, ${ }^{1}$ Nikolaos Lazaridis*, 'Nikolaos Koukias, ${ }^{1}$ Andrea Telese, 'Deborah Costa, ${ }^{1}$ Claudia Coppo, ${ }^{1,2}$ Yoshikazu Hayashi, 'Edward I Despott. 'The Royal Free Unit for Endoscopy, The Royal Free Hospital And University College London (UCL) Institute For Liver And Digestive Health, London, UK; ${ }^{2}$ Division of Gastroenterology, Department of Medicine, Jichi Medical University, Shimotsuke, Japan

\subsection{6/gutjnl-2019-BSGAbstracts.95}

Introduction Small bowel diverticula are a rare cause of gastrointestinal (GI) bleeding. Their pathogenesis is still unclear and can be found in up to $1-2 \%$ of the general population. Although these lesions are usually asymptomatic, mid-gut bleeding from diverticula in the jejunum or ileum could lead to a life-threatening situation, warranting emergency invasive therapy and often abdominal surgery.

Aims and Methods Our aim was to demonstrate the usefulness of the double-balloon enteroscopy (DBE) in the setting of an acute, severe small bowel diverticular bleed. A 79-year-old woman with hypertension and type II diabetes mellitus was referred to our institution with melaena and severe anaemia requiring urgent, repeat blood transfusions. Bidirectional conventional endoscopy did not reveal the cause of bleeding. Small bowel capsule endoscopy (SBCE) showed multiple diverticula within the jejunum and ileum. Emergency computed tomography (CT) mesenteric angiography demonstrated a faint 'blush' at one of the jejunal lesions.

Results Once the patient $\mathrm{w}$ as haemodynamically stable, emergency anterograde DBE was performed under general anaesthesia (GA) in our main operating theatres. The enteroscope was inserted into the jejunum, approximately 1.5 meters post- pylorus. The culprit cause of the bleeding was identified within a large $(5 \mathrm{~cm}$ orifice $)$ diverticulum, where a large, adherent, pulsating blood clot was seen. In the first instance, peri-lesion, quadrantic injection of a total of $20 \mathrm{mls}$ of adrenaline solution ( 1 in 10,000 dilution) was performed. The clot was then cautiously removed with a long endoclip to reveal the actively bleeding vessel which was then promptly clipped. A total of 3 clips were placed for effective haemostasis and a submucosal tattoo was placed adjacent to the bleeding point for future reference. The patient remained stable after the procedure and did not require any further blood transfusion.

Conclusion DBE facilitated endotherapy is a precise, safe and minimally invasive approach to the effective management of severe bleeding caused by small bowel diverticula.

\section{PTH-071 COMBINED ENDOSCOPIC CLOSURE OF COLOVESICAL FISTULA}

Faisal Nawaz*, Hemant Ojha, Monzur Ahmed. Good Hope Hospital, Birmingham, UK

\subsection{6/gutjnl-2019-BSGAbstracts.96}

Introduction Colovesical fistulas (CVFs) occur as a complication of diverticulitis, cancer, or Crohn's disease. The sigmoid colon is the most frequently involved segment. The most common presenting symptoms are pneumaturia and dysuria, followed by faecaluria, abdominal pain and, rarely, haematuria. The diagnosis is usually made clinically but can be confirmed by cystoscopy, sigmoidoscopy, barium enema, CT scan, MRI, or virtual colonoscopy. The usual management for symptomatic patients is colonic resection (which can be performed laparoscopically) but this approach is associated with morbidity and mortality. Only a few cases of endoscopic management by clip closure, using through-the-scope (TTS) or over-the-scope (OTS) clips have been described in the literature. Here, we present a case of a CVF, which was managed with multiple TTS clips, thus avoiding surgical intervention.

Methods A frail 84 year old man had a background history of coronary artery bypass graft, aortic valve replacement (tissue valve), dialysis dependent chronic renal failure and sigmoid diverticular disease. He was investigated for pneumaturia and recurrent urinary tract infections 12 months ago and was diagnosed on CT scan as having CVF secondary to sigmoid diverticular disease. He developed faecaluria, He was referred by the colorectal surgeon for endotherapy as he was considered too frail for any surgical intervention. A combined simultaneous cystoscopy and flexible sigmoidoscopy (using a gastroscope) was undertaken under conscious sedation. The cystoscopy identified a fistula in the dome of the bladder (on left side). The sigmoid colon was carefully examined and revealed diverticular disease but no obvious fistula was visible. Hence, indigocarmine solution (16 mg/litre), warmed to body temperature, was instilled into the bladder via the cystoscope and the blue solution was eventually seen to emerge in the sigmoid colon through the fistula. This site was marked with a Resolution 360 clip (Boston Scientific). After this, 4 further clips were placed onto the fistula in order to close it. Finally, the site was marked with a tattoo, in case a repeat procedure was needed in the future.

Results The procedure took almost two hours and was successfully performed. The patient was discharged on the same day. 